\title{
Nonlinear Finite Element Analysis and Optimum Design of Spot-Welded Joints and Weld-Bonded Joints
}

\author{
Shengyong Zhang \\ Department of Mechanical and Civil Engineering, College of Engineering and Sciences, Purdue University Northwest, \\ Westville, USA \\ Email: syzhang@pnw.edu
}

How to cite this paper: Zhang, S.Y. (2020) Nonlinear Finite Element Analysis and Optimum Design of Spot-Welded Joints and Weld-Bonded Joints. Journal of Applied Mathematics and Physics, 8, 1715-1728. https://doi.org/10.4236/jamp.2020.89129

Received: July 20, 2020

Accepted: August 31, 2020

Published: September 3, 2020

Copyright () 2020 by author(s) and Scientific Research Publishing Inc. This work is licensed under the Creative Commons Attribution International License (CC BY 4.0).

http://creativecommons.org/licenses/by/4.0/ (c) (i) Open Access

\begin{abstract}
Resistance spot welding and hybrid weld bonding have wide applications in the body work construction within the automobile industry. The integrity of the spot welds and applied adhesives determines the body assembly rigidity and dynamic performance. Incorporating contact nonlinearity and geometric nonlinearity, finite element analysis (FEA) have been carried out to investigate the structural stiffness and strength of both spot-welded and weld-bonded assemblies. Topology optimization has been performed to reveal the distributions of material effectiveness in the overlap regions and suggest a feasible method for removing underutilized material for weight reduction. Design optimization has been conducted with an aim to reduce the maximum von Mises stress in the assembly to minimum by choosing optimum values for a set of design variables, including the weld spacing, weld diameter and overlap width.
\end{abstract}

\section{Keywords}

Finite Element Analysis, Optimum Design, Spot-Welded Joint, Weld-Bonded Joint, Lightweight Design

\section{Introduction}

Spot welding is a quick welding process and can be easily adapted for automation when combined with robots and control systems. The weld-bonded joining is a technology in which both adhesives and spot-welds are utilized to fasten metal sheets. Spot-welding and weld-bonding have been the conventional joining technologies for assembling metal sheets together for decades, notably for 
vehicle body construction. A modern automobile has its body structure comprised of stamped panels fastened with several thousands of spot welds and adhesive bonding. The integrity of the spot welds and the applied adhesives determines the body stiffness and dynamic performance. Higher body stiffness, including both the bending stiffness and torsional stiffness, is deemed to be better by designers. Body stiffness has an impact on the automotive NVH parameters as well. For example, the annoying squeak and rattle noise resulting from the relative motion between joined components indicates a poor quality of an automotive body structure.

Murray [1] studied the loaded behavior of spot-welded thin-walled structures from the perspective of vehicle crashworthiness. Experimental testing on transverse joints in the floorpans demonstrated the effects of the spot weld pattern on the energy absorbing capacity of the joints. Thin-walled tubular members fastened with spot welds were investigated to study the influence of the spot weld spacing on the energy absorbing capacity as well. Deng, Chen and Shi [2] worked on spot welds by 3D FEA. Detailed finite element results were obtained for spot-welded specimen with different sheet thickness to spot-weld diameter ratios. The FEA results agreed with published experimental observations. For typical vehicle structure jointed by several thousands of spot welds, it is formidable to analyze the influence of each and every spot weld on the load transfer and structural rigidity. Salvini, Vivio and Vullo [3] put forward a spot weld element for structural modeling. Each spot weld was simulated by a rod element and each end of the rod was joined to the metal sheet through a set of beam elements. Numerical examples demonstrated the advantage of the new element on improving the result accuracy without mesh refinement. High-strength steel (HSS) has found more and more applications in the construction of vehicle body structures. Tanegashimaa, Akebonoa, Katoa et al. [4] conducted experimental testing on thin-walled, spot-welded members made of HSS. The growing of cracks in the spot-welded joints was observed to study the fatigue properties when the joints were subjected to variable loads. Dhawaleb and Ronge [5] developed a mathematical model to predict the tensile shear strength of spot-welded joints using the design and process parameters. Three design variables and two process variables were involved in the developed model. Experimental testing was conducted for validation of the analytical results.

Both experimental and numerical studies on the weld-bended joints have been carried out since it was developed. Finite element analysis was carried out in references [6] [7] to study the structural performance of weld-bonded joints. The stress distributions in the lap zone of a single-point lap weld-bonded joint were calculated and compared with those of spot-welded joint and adhesive-bonded joint. It was found that the stresses in the weld-bonded joints were low and uniform. The stress concentration that occurred around the nugget in spot-welded joints was avoided, leading to a better fatigue performance. It was concluded that the tearing strength of weld-bonded joints was superior to that of adhesive-bonded 
joints because of the existence of spot-welds. Testing results illustrated the load distribution in the lap zone corresponding to different elastic modulus of adhesives. It was concluded that application of adhesive with large elastic modulus will help uniform stress distribution. Fujiia, Tohgo, Suzukib, et al. [8] compared spot-welded joints and weld-bonded joints on their fatigue strength. Strain was measured to study the development and propagation of fractures in the applied adhesive. It was concluded that the cracks likely initiated at the edge of overlap region in adhesive bonded joints. Almeida, Camilo, Silva et al. [9] conducted numerical and experimental studies on three different adhesives and compared with each other on the aspect of joint strength. It was concluded that a weld-bonded joint enhanced strength, stiffness, and peel resistance than that of a spot-welded joint.

FEA-based topology optimization and design optimization have been performed for spot-welded joints and weld-bonded joints in this study. FEA is conducted in which the nonlinear responses due to sheet contacting and large displacement are involved. Topology optimum results reveal the distributions of material effectiveness in the overlap regions and suggest a feasible method for removing underutilized material for weight reduction. Design optimization has been conducted with an aim to reduce the maximum von Mises stress in the assembly to minimum under specified constraints.

\section{Spot-Welded Joints}

\subsection{Finite Element Analysis with Contact Nonlinearity}

Figure 1 shows an assembly constitutes of two panels joined together using a single spot weld. Both panels are made of A366 steel and each one is measured to be $120 \mathrm{~mm}$ long, $15 \mathrm{~mm}$ wide and $1 \mathrm{~mm}$ thick. The weld nugget has a diameter of $6 \mathrm{~mm}$ and is located at the center of the overlap region, which is $20 \mathrm{~mm}$ in the longitudinal direction. It is assumed there is a $0.2 \mathrm{~mm}$ gap between the two metal sheets. The material modulus of elasticity and Poisson's ratio are set to be 190 GPa and 0.25 for the metal sheets, and $200 \mathrm{GPa}$ and 0.2 for the weld nugget, respectively.

Figure 2 shows the FE model of the spot-welded assembly. The sheets are meshed with solid finite elements. Each element has eight nodes and each node has three translational degrees of freedom. The weld nugget is meshed with solid elements too by using volume sweep meshing. The sweep meshing method starts by meshing a source area with, for example, 2D quadrilateral elements. Sweeping these $2 \mathrm{D}$ elements will generate layers of hexahedral elements in an existing volume between the source and target area. The assembly is to be clamped at one end and loaded with a uniform distributed force at the other end. Because of its symmetric configurations, it is proper to model only half of the assembly by applying symmetric constraints. See Figure 2(a). The assembly is meshed nonuniformly, with finer meshing in the overlap region and coarse meshing away from that region as shown in Figure 2(b). 


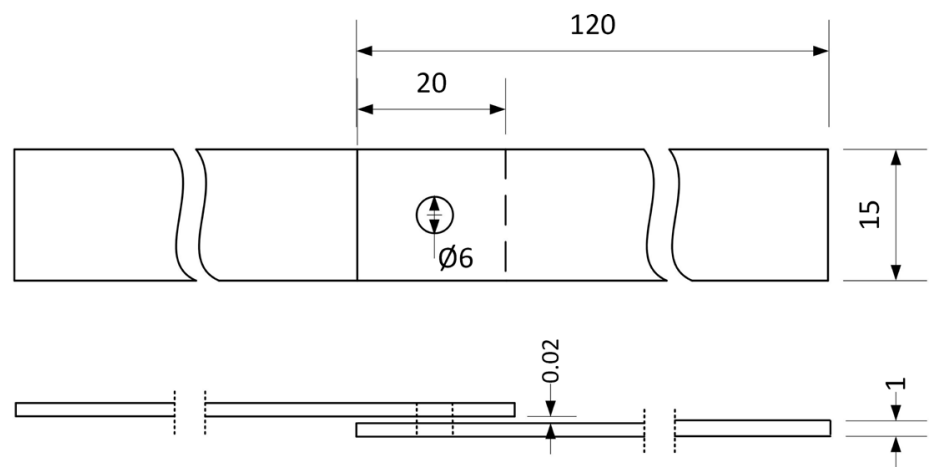

Figure 1. Front and top view of a spot-welded assembly. All dimensions are in millimeters.
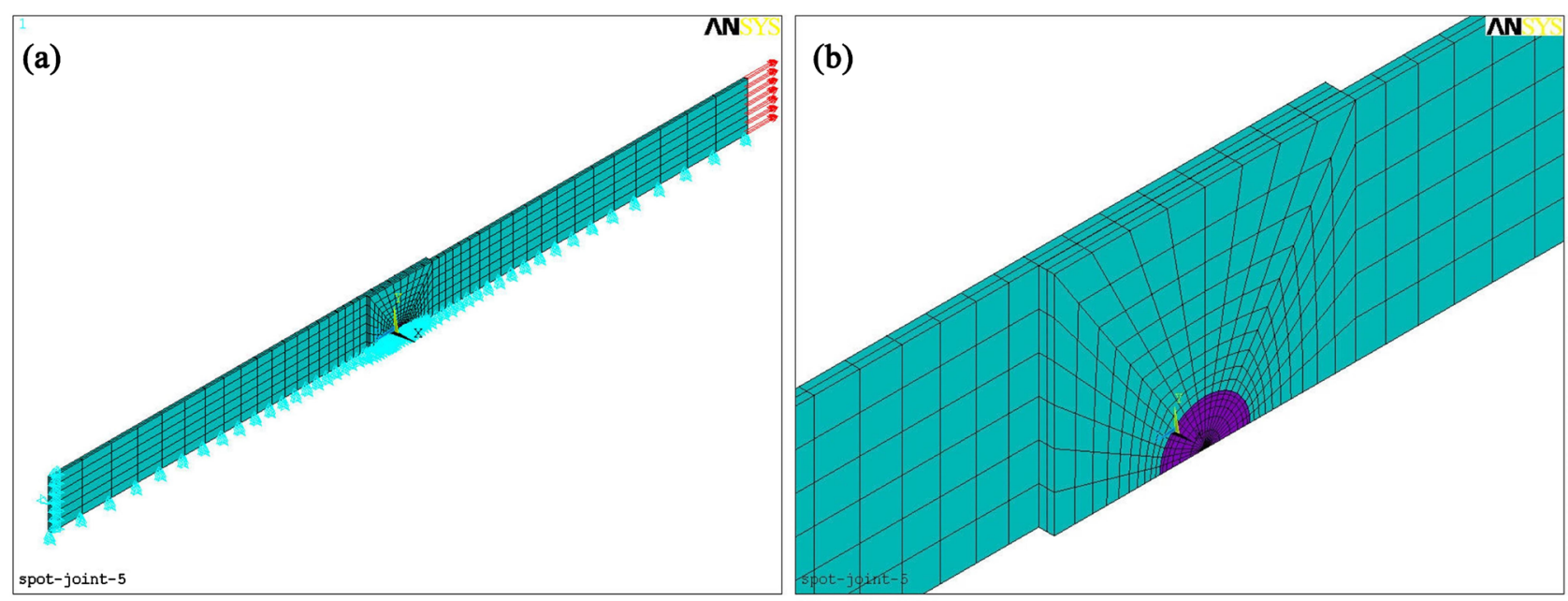

Figure 2. FE model of the spot-welded assembly. (a) Symmetric constraints applied along the longitudinal direction; (b) Fine meshing in the overlap zone and coarse meshing outside the overlap zone.

Figure 3(a) shows the deformation of the assembly under specified loadings. It is clear that the two sheets come into contact and interfere with each other in the overlap region. The existing contact and interference will result in unreliable FEA results, including the assembly compliance and strength. It suggests that the nonlinearity due to the contacting surfaces should be involved in the FEA for accurate simulation of structural performance. Surface contacting is a process involving boundary nonlinearity which is too complicate to be analyzed with analytical models. Many researches rely on numerical methods for approximating this nonlinear contacting process. In this study, 3D flexible-flexible, surface contact elements are utilized to prevent inter-penetration of sheets during the deformation process. Figure $3(\mathrm{~b})$ shows the deformation of the assembly under the same prescribed loadings. Compared to Figure 3(a), it is clear that the inter-penetration between the two sheets has been prevented with the application of the contact pair elements.

\subsection{Topology Optimization}

Figure 4 shows the contour plot of von Mises stress in the overlap region. The 


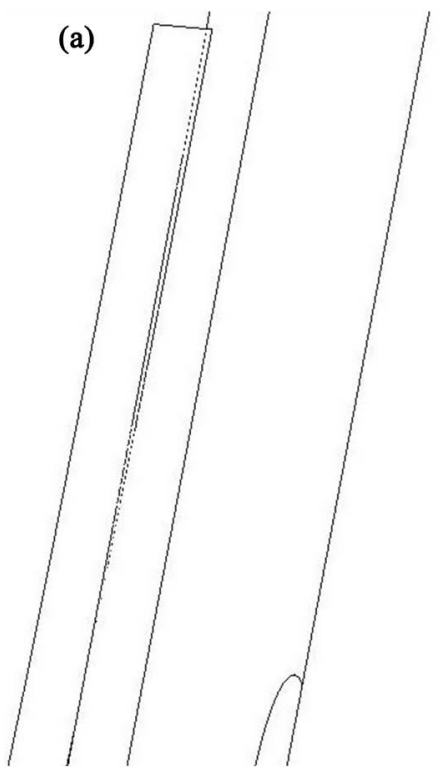

(b)

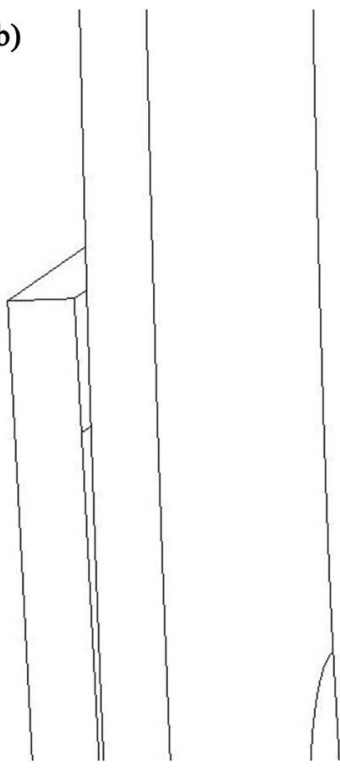

Figure 3. Deformations of the assembly under specified loads. (a) Boundary nonlinearity neglected; (b) Boundary nonlinearity embodied.

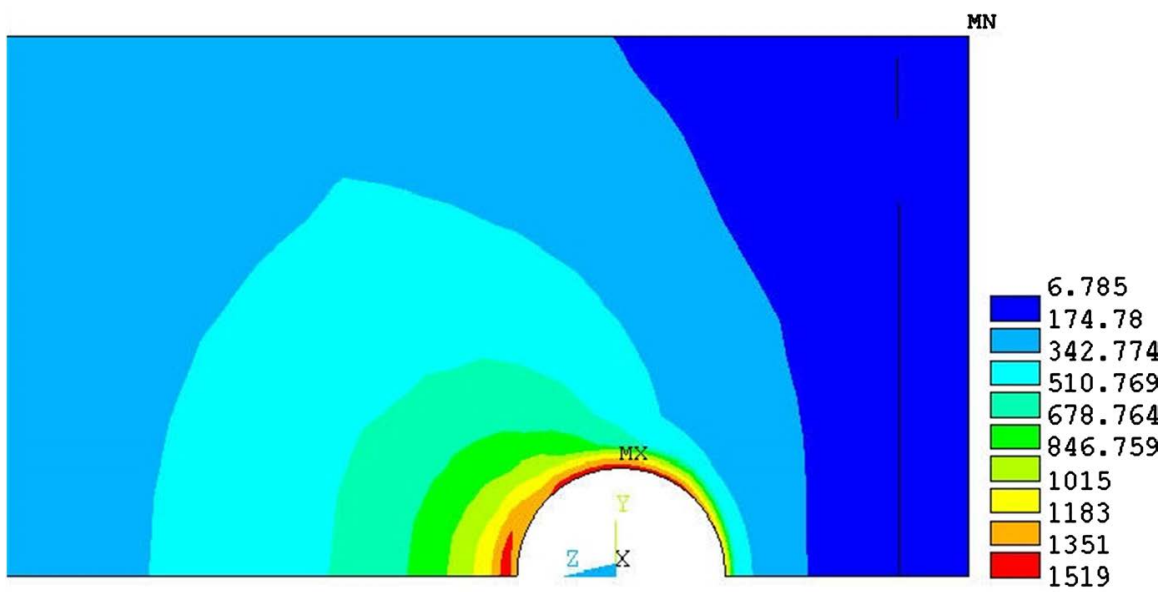

Figure 4. Contour plot of von Mises stress over the lap zone.

stress has a nonuniform distribution and the stress nonuniformity provides an insight of the material effectiveness in the overlap region. It is interpreted that material in the portions where stress values are low is less effective in usage. Removal of material from these portions will help lessen the stress nonuniformity and as a result, improve the effectiveness of the material over the entire overlap region. It is also clear the stress concentration occurs at the periphery of the weld nugget, illustrating that cracks are most likely developed around the nugget and leading to a fracture failure in the vicinity. To show the stress changes in more details, Figure 5(a) and Figure 5(b) plot the variations of the von Mises stress and shear stress, respectively, over the lap zone from the left end to the right end. Both have their peak values at either side of the weld nugget. It illustrates that fractures usually initiate near the weld nugget in the spot-welded assembly. 


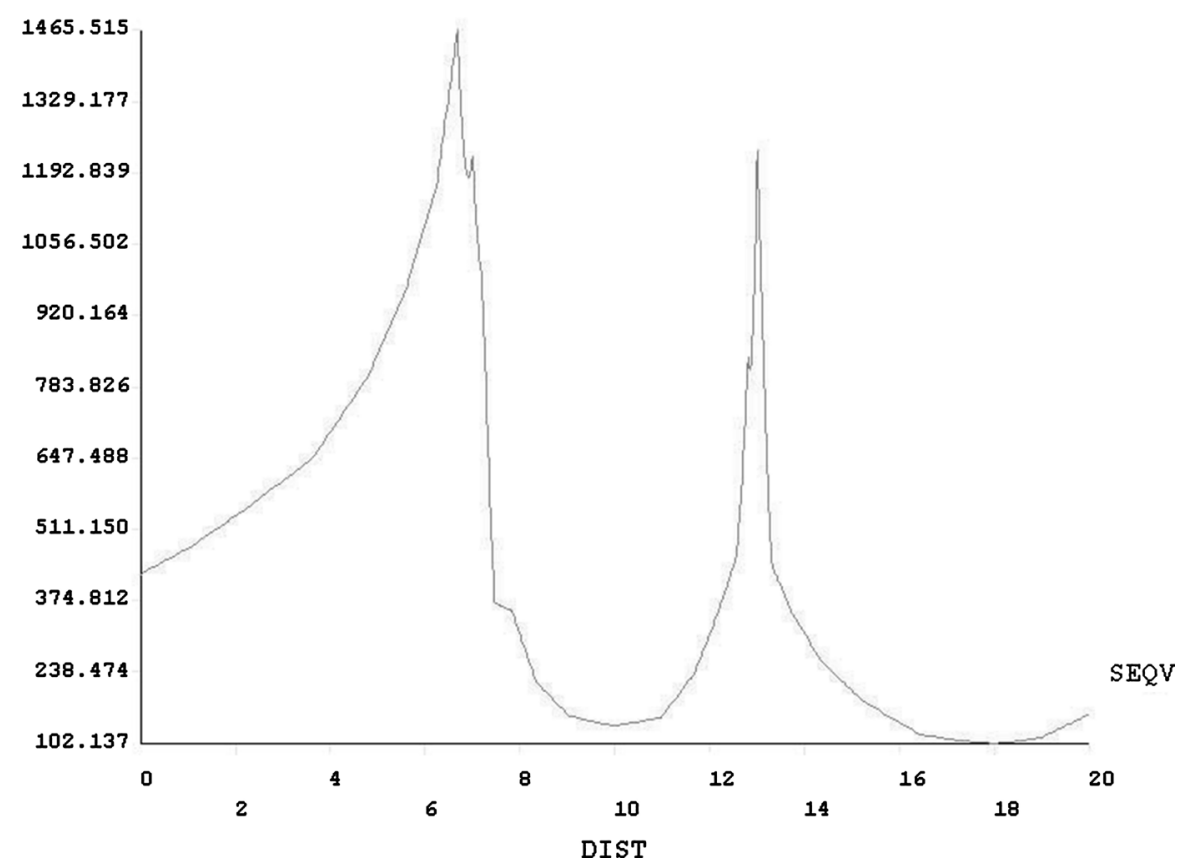

(a)

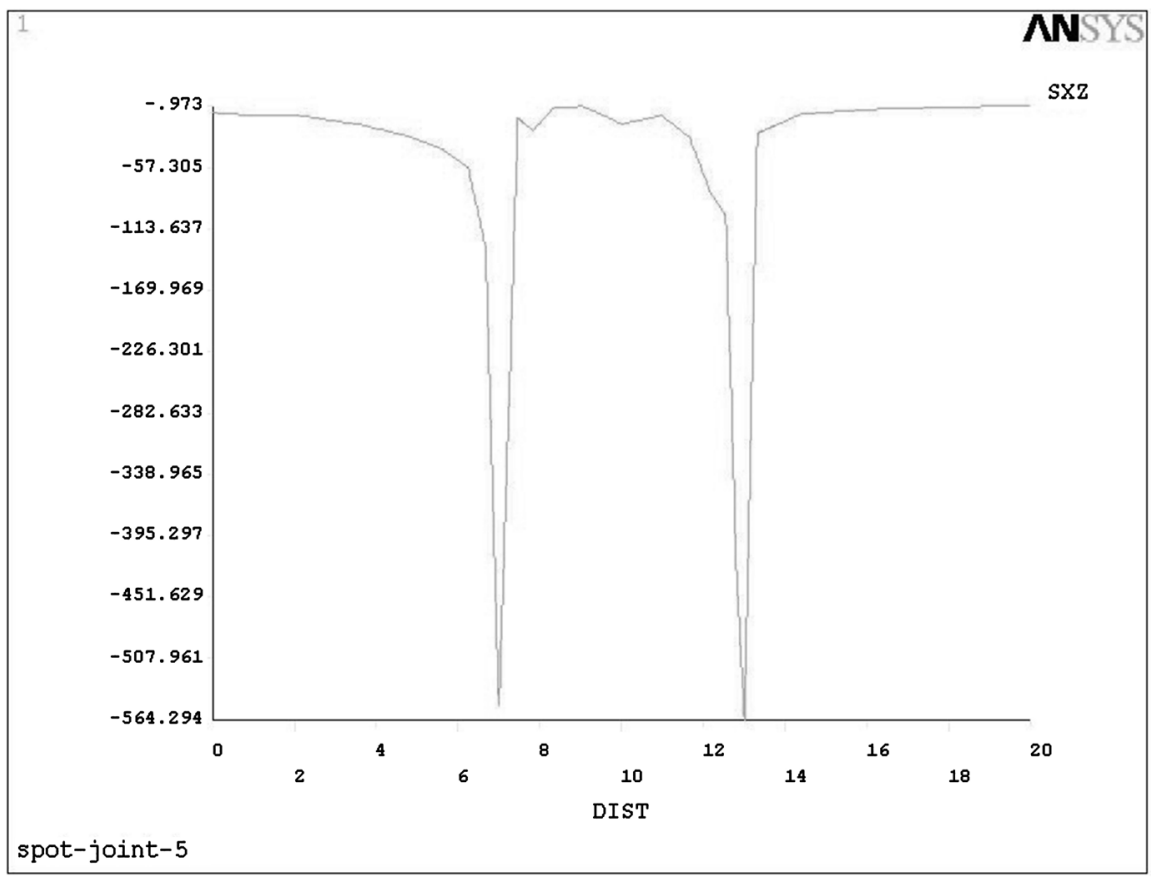

(b)

Figure 5. Path plots of the stresses over the lap zone. (a) von Mises stress; (b) Shear stress.

Topology optimization is based on the homogenization method for redistributing material rationally in the design domain. In this research, the assembly's compliance, which is inversely proportional to its stiffness, is set to be the objective function. A $20 \%$ weight reduction for the metal sheets is specified as the constraint. The overlap zone of the assembly is defined as the design area, while all finite elements outside of the overlap zone are regarded as dummy elements 
in the optimization process.

Figure 6(a) plots the optimization results for the assembly in terms of density functions that range from 0 to 1 . Elements with low density values are less stressed and material contained in these elements is correspondingly less effective in sustaining the external loads. Reducing the assembly weight by removing the material with low effectiveness from the design domain has relatively fewer negative effects on the assembly stiffness. Figure 6(b) modifies the above optimum results by making the elements with low density values invisible. One can smooth the resulting rough boundary by FEA interpreting algorithm for better sense of appearance. In conclusion, optimum results reveal the material effectiveness in the design area and help designers remove material rationally from the overlap zone. Similar optimization results for an assembly with multiple weld nuggets are illustrated in Figures 7(a)-(d). Figure 7(a) shows the FEA model of the assembly with five spot welds. Figure $7(\mathrm{~b})$ plots the von Mises stress distribution. Figure 7 (c) shows the distribution of the density functions ranging from 0 to 1 in the overlap zone, which is defined as the design area in the optimization process. It is interpreted again that the material in the portions where the density values are low is less effective and can be removed with relatively few adverse influences on the structural stiffness, see Figure 7(d).

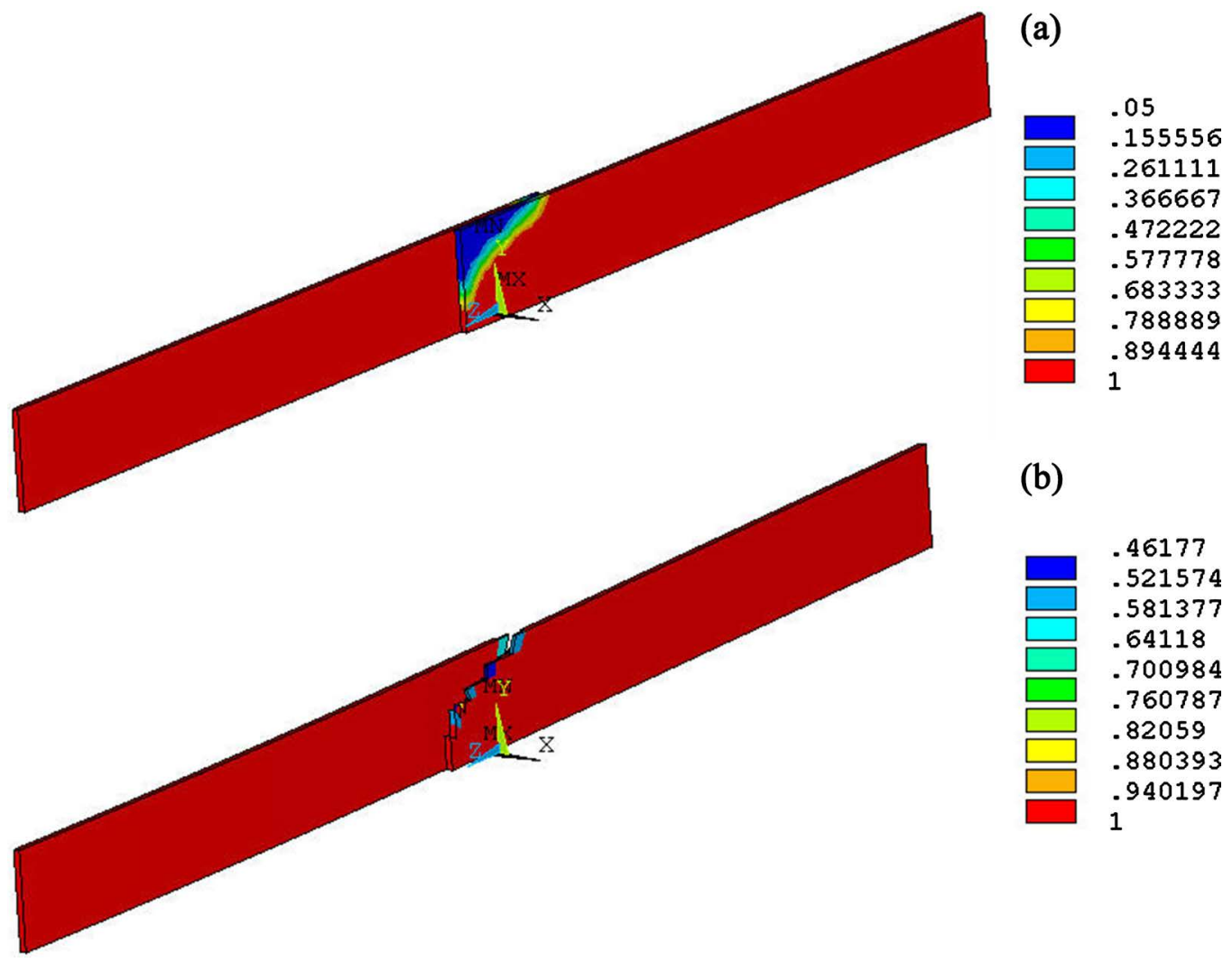

Figure 6. Topology optimization in the overlap zone. (a) Distribution of density functions ranging from 0 to 1 ; (b) Optimum results by removing less stressed material. 

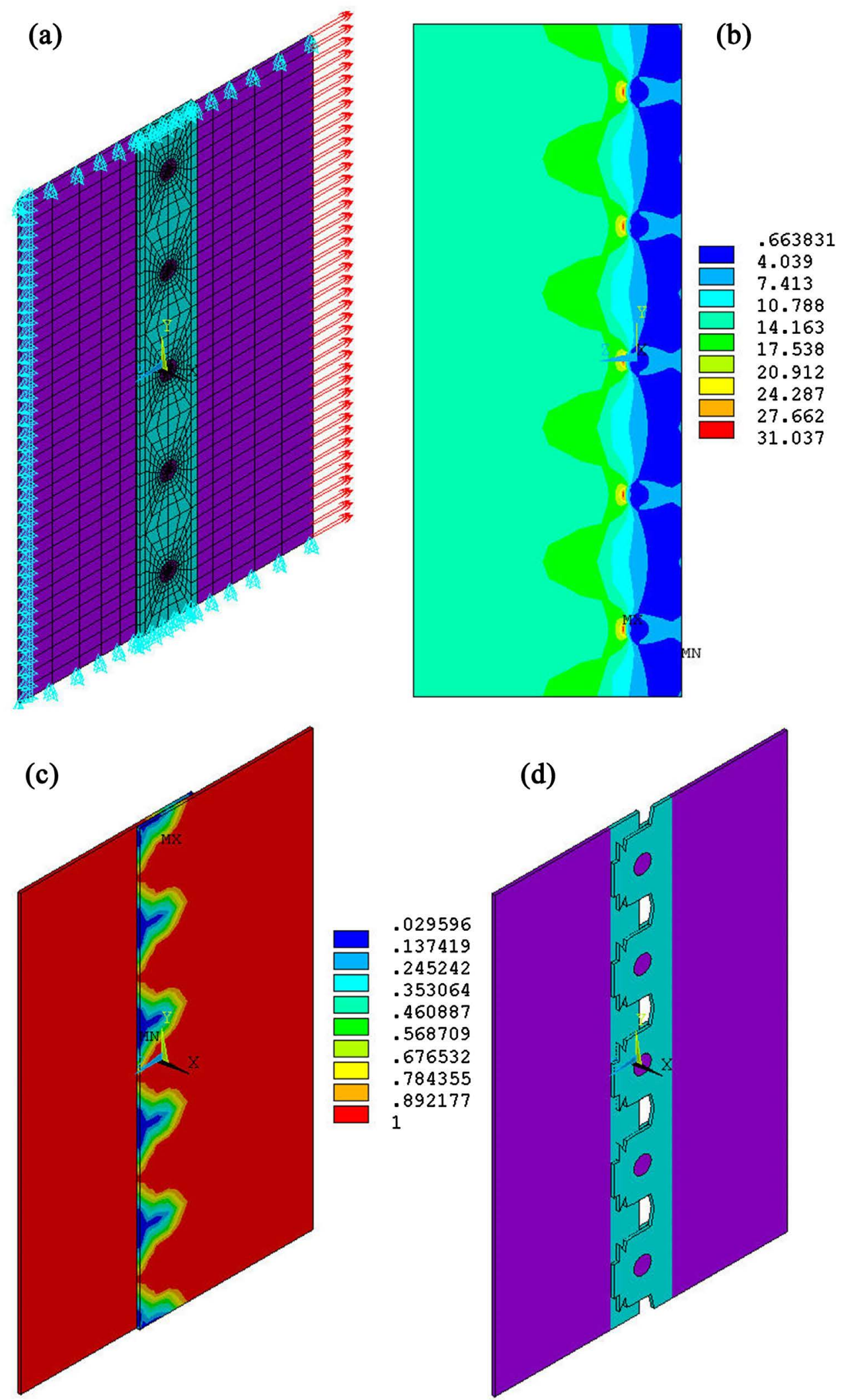

Figure 7. Optimum results of an assembly with five spot welds. (a) FE model; (b) von Mises stress distribution; (c) Density function distribution; (d) Optimum results by removing less stressed material. 


\section{Weld-Bonded Joints}

\subsection{Finite Element Analysis with Geometric Nonlinearity}

The weld-bonded joint investigated in this study is the same in its dimensions as the spot-welded one shown in Figure 1, except of the adhesive bonding layer between the two joined metal sheets in the overlap region. The adhesive layer is $0.2 \mathrm{~mm}$ thick and its Young's modulus and Poisson's ratio are $2875 \mathrm{MPa}$ and 0.42 , respectively.

Figure 8 shows the FE model of the weld-bonded assembly in which the two sheets are meshed with solid elements and the thin, in-between adhesive layer is meshed with shell elements. Shell elements have six DOFs at each node, while solid elements have only three translational DOFs at each node. The DOFs of the shell elements and solid elements have been coupled to avoid hinge connections in the assembly. The DOFs of the shell elements are set to be master DOFs while the DOFs of the solid elements the slave DOFs. The established couplings enable

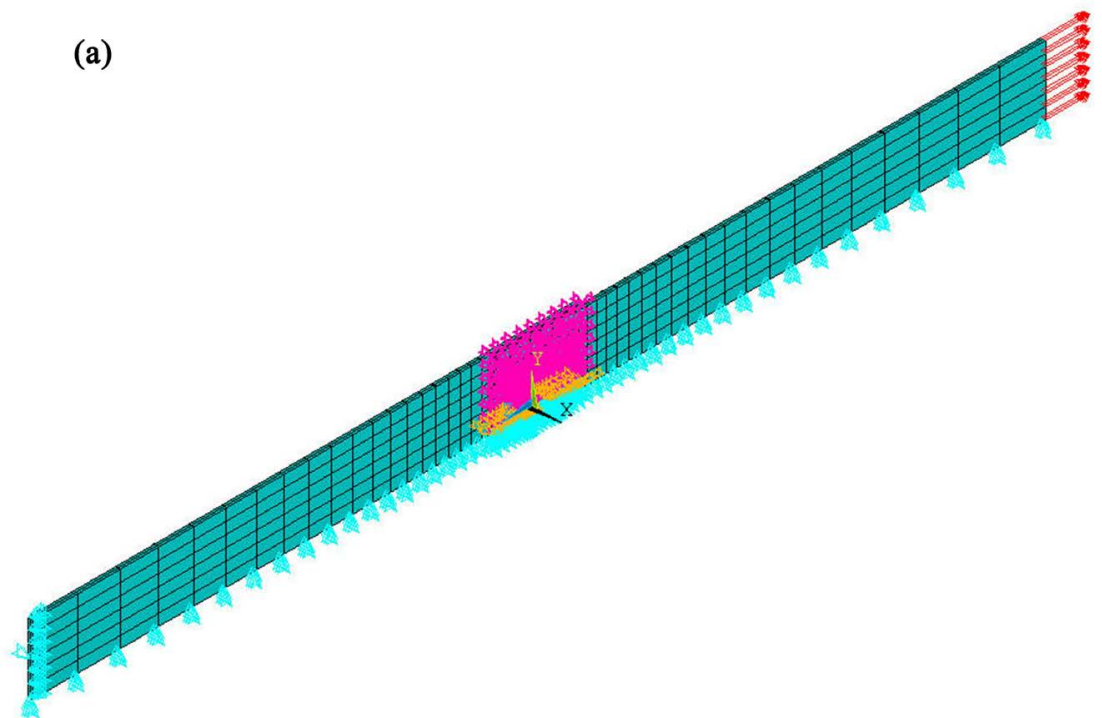

(b)

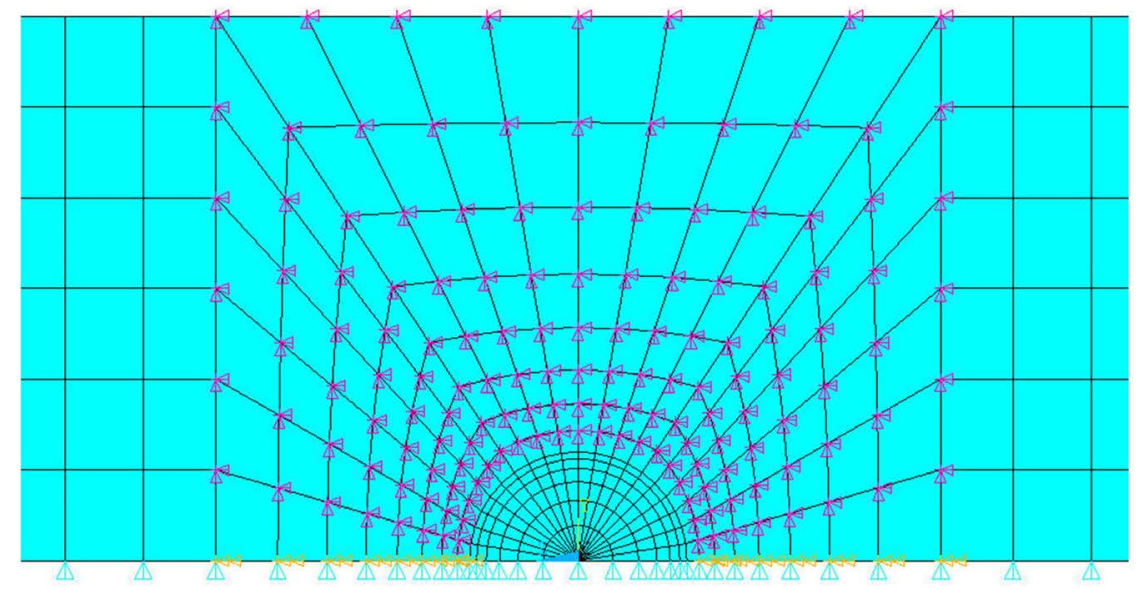

Figure 8. FE model of weld-bonded assembly. (a) Symmetric constraints applied along the longitudinal direction; (b) Couplings between shell elements and solid elements. 
the transfer of moment between the two different types of finite elements in the assembly. The weld-bonded assembly is constrained and loaded in the same way as that for the spot-welded assembly.

Figure 9 shows the assembly is experiencing a large displacement under the specified loading. Large displacement will incur geometric nonlinearity in the responses, resulting in a reduction in the assembly stiffness. The von Mises stress is plotted in Figure 10. Figure 11(a) and Figure 11(b) plot the variations of von Mises stress and shear stress, respectively, over the lap zone from the left end to the right end. The two peaks correspond to either side of the weld nugget. Being compared to the stress distributions of the spot-welded assembly, the stress nonuniformity has been mitigated in the overlap zone of the weld-bonded assembly. For example, the variation of von Mises stress is calculated to be about $95 \%$ in the pure spot-weld assembly, in which the percentage value is computed based on the max. and min. stress values in the overlap region. In the weld-bonded assembly, however, this percentage value is reduced to be approximately $82 \%$.

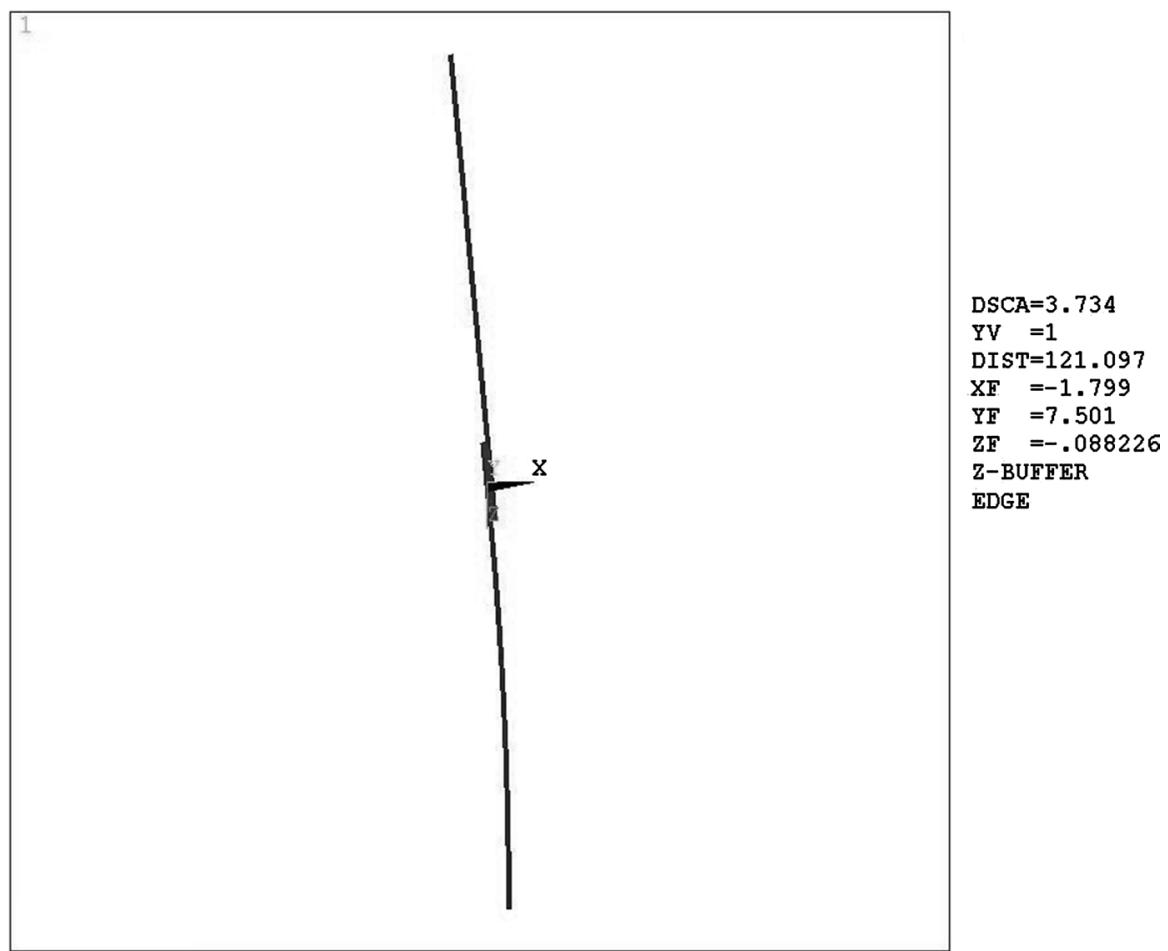

Figure 9. Geometric nonlinearity due to large displacement.

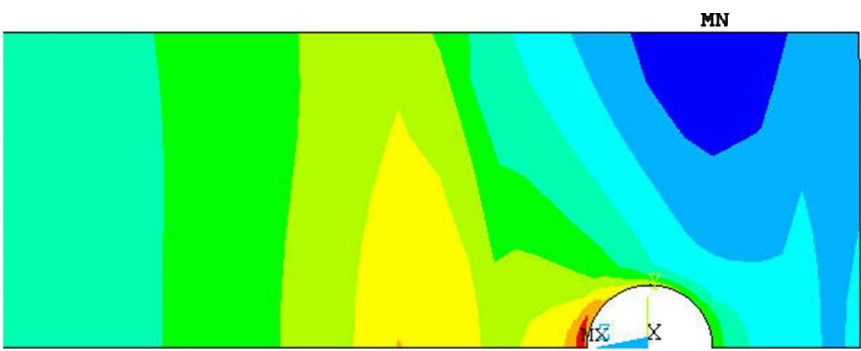

Figure 10. Contour plot of von Mises stress over the lap zone. 


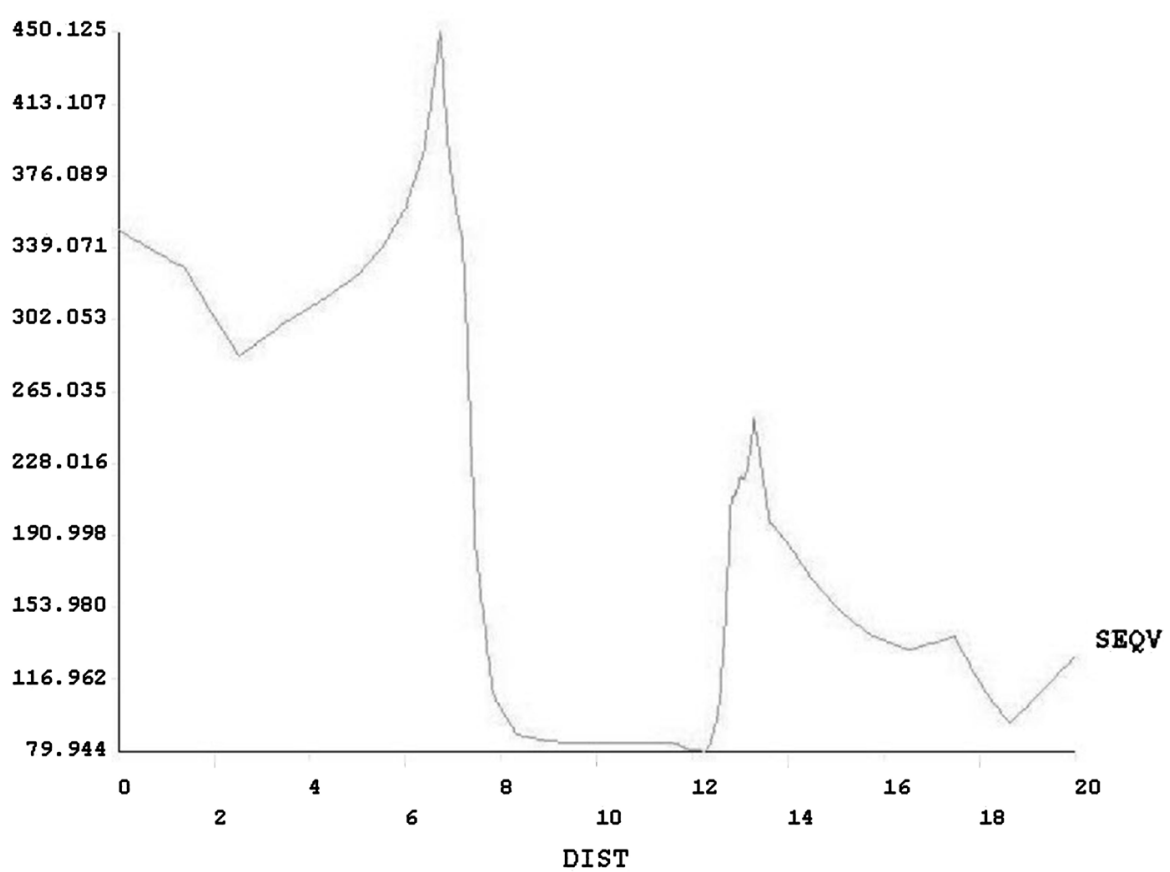

(a)

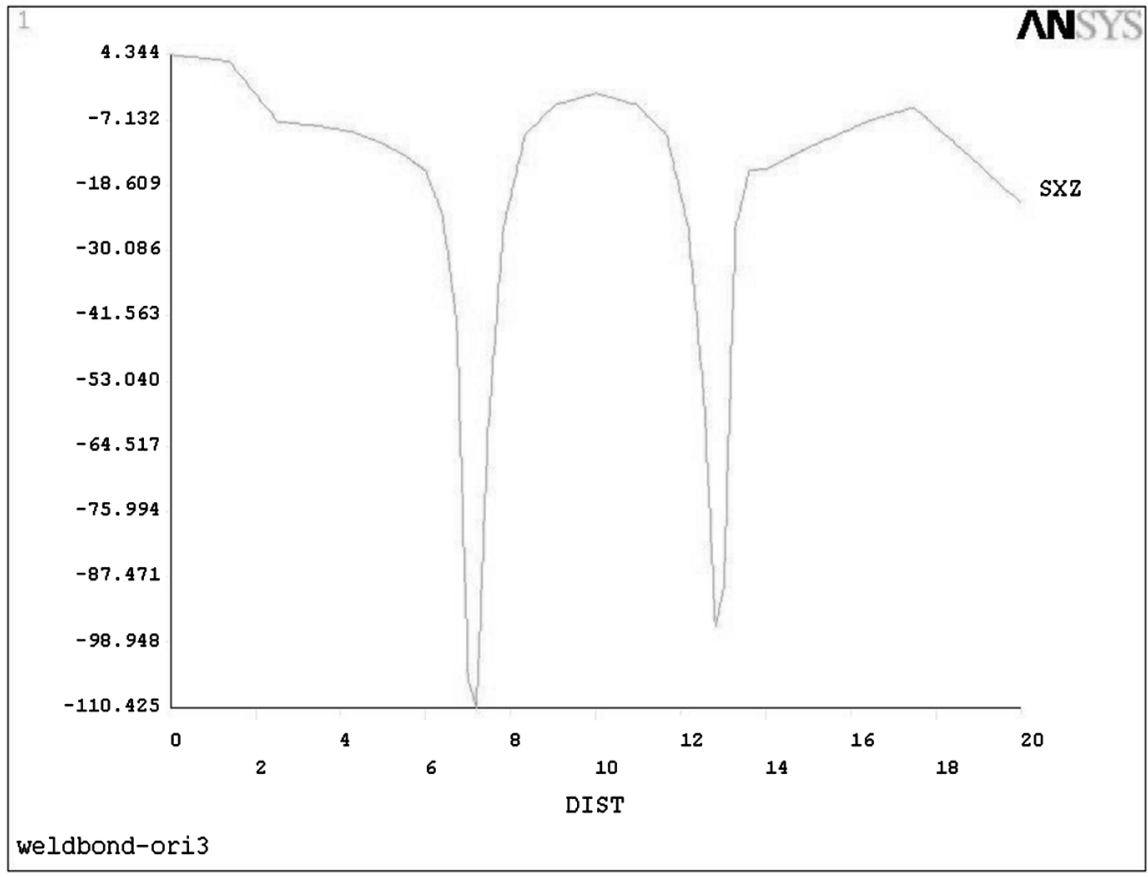

(b)

Figure 11. Path plots of the stresses across the overlap zone. (a) von Mises stress; (b) Shear stress.

Except of the mitigation in the stress nonuniformity, the maximum stress values in the vicinity of the nugget has been significantly decreased in the weld-bonded assembly as well. In comparison with the stress values shown in Figure 5, it is clear that the maximum values of both the von Mises stress and shear stress have been significantly reduced. As a consequence, the fatigue performance of the 
weld-bonded assembly has been notably improved.

\subsection{Design Optimization}

The integration of spot welding and adhesive bonding affects the load bearing capability of a weld-bonded assembly. There are dozens of parameters that should be considered in the design and fabrication of weld-bonded structures. For example, the adhesive related parameters (adhesive elastic modulus, adhesive thickness, etc.), the weld related parameters (welding time, welding current, weld diameter, weld spacing, etc.), and the overlap region related parameters (overlap length and width, surface treatment, etc.). A series of FEA analysis has been conducted to investigate the variations of the assembly stiffness with respect to the changes in the weld spacing and the overlap width. The assembly stiffness is simply defined as the applied force divided by the corresponding displacement in this study. The FE model is the same as the spot-welded structure shown in Figure 7(a), except of the applied adhesive ( $=2875 \mathrm{MPa}$ and $\mu=$ $0.42)$ in the overlap region between the two sheets. The weld diameter (d) is set to be $6 \mathrm{~mm}$. To study the variations of the assembly stiffness to changes in the weld spacing (S) and overlap width (L) over a wide range, the dimensions of the weld spacing is selected to be $20 \mathrm{~mm}, 30 \mathrm{~mm}$ or $40 \mathrm{~mm}$, and the overlap width is $16 \mathrm{~mm}$ or $24 \mathrm{~mm}$, respectively. Nonlinear FEA is conducted and Figure 12 plots the changes in the assembly stiffness with respect to $\mathrm{S} / \mathrm{d}$ and L/d in the specified ranges.

Design optimization aims at achieving the best of all possible designs by maximizing or minimizing the objective function and meeting the prescribed constraints on a set of design variables. It ensures to, for example, maximize the structural stiffness, minimize the stress in the structure, or optimize dynamic performance of the structure. At the same time, all constraints and limitations prescribed for the design, such as the weight, cost, dimensions, and other design variables, are to be satisfied in the optimization process. Design optimization has been a key engineering methodology for designing a mechanical component in an optimum manner. Optimum design for the weld-bonded assembly has been carried out based on the above FEA. The maximum von Mises stress in the overlap

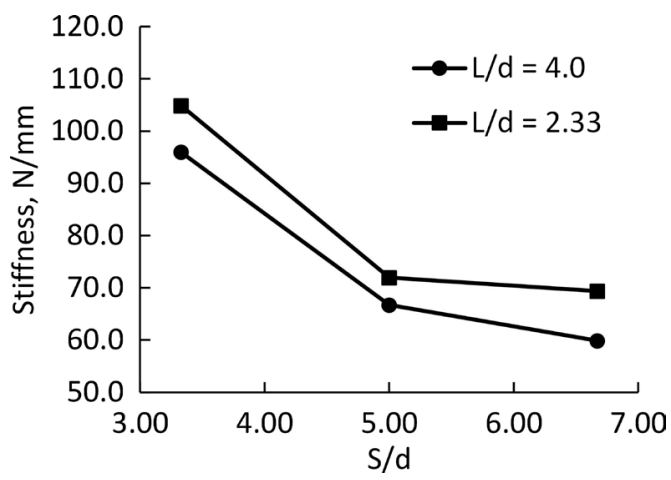

Figure 12. Variations of assembly stiffness with respect to the weld spacing and overlap width. 
region is targeted as the objective function for minimization, while the design variables as set to be the weld spacing, overlap width and weld diameter. In the optimization process, the limited dimensions of the weld spacing range from 20 $\mathrm{mm}$ to $40 \mathrm{~mm}$, the overlap width range from $15 \mathrm{~mm}$ to $30 \mathrm{~mm}$, and the weld diameter range from $5 \mathrm{~mm}$ to $7 \mathrm{~mm}$. It is concluded that the max. von Mises stress has been reduced to $61.8 \mathrm{MPa}$ corresponding to optimum values of the weld spacing, overlap width and weld diameter to be $31.64 \mathrm{~mm}, 27.71 \mathrm{~mm}$, and $5.91 \mathrm{~mm}$, respectively.

\section{Conclusion}

Resistance spot welding and hybrid weld bonding are commonplace technologies for assembling thin-walled metal sheets. FEA-based topology optimization and design optimization have been carried out for spot-welded and weld-bonded assemblies in this study. Nonlinear FEA has incorporated the contact nonlinearity and geometric nonlinearity in the simulation of assembly responses. Topology optimum results demonstrate the distributions of material effectiveness in the overlap region and provide the designer an insight to remove material properly for reducing assembly weight. Design optimization has been conducted with an aim to minimize the maximum von Mises stress in the assembly under specified constraints. Optimum values are determined for a set of design variables, including the weld spacing, weld diameter and overlap width.

\section{Conflicts of Interest}

The author declares no conflicts of interest regarding the publication of this paper.

\section{References}

[1] Murry, N.W. (1994) Load Behavior of Thin-Walled Spot-Welded Structures Used in the Vehicle Industry. Engineering Structures, 16, 363-367. https://doi.org/10.1016/0141-0296(94)90029-9

[2] Deng, X., Chen, W. and Shi, G. (2000) Three-Dimensional Finite Element Analysis of the Mechanical Behavior of Spot Welds. Finite Element in Analysis and Design, 35, 17-39. https://doi.org/10.1016/S0168-874X(99)00053-0

[3] Salvini, P., Vivio, F. and Vullo, V. (2000) A Spot Weld Finite Element for Structural Modeling. International Journal of Fatigue, 22, 645-656. https://doi.org/10.1016/S0142-1123(00)00044-X

[4] Tanegashimaa, R., Akebonoa, H., Katoa, M. and Sugetaa, A. (2011) The Influence of the Load Variation on Fatigue Strength of the Spot-Welded Joint Using the High Strength Steel. Procedia Engineering, 10, 3405-3410. https://doi.org/10.1016/j.proeng.2011.04.561

[5] Dhawaleb, P.A. and Ronge, P. (2019) Parametric Optimization of Resistance Spot Welding for Multi Spot Welded Lap Shear Specimen to Predict Weld Strength. Materials Today: Proceedings, 19, 700-707. https://doi.org/10.1016/j.matpr.2019.07.756

[6] Chang, B.H., Shi, Y.W. and Dong, S.J. (1999) Comparative Studies on Stresses in Weld-Bonded, Spot-Welded and Adhesive-Bonded Joints. Journal of Materials 
Processing Technology, 87, 230-236.

https://doi.org/10.1016/S0924-0136(98)00355-0

[7] Chang, B.H., Shi, Y.W. and Dong, S.J. (2000) Studies on a Computational Model and the Stress Field Characteristics of Weld-Bonded Joints for a Car Body Steel Sheet. Journal of Materials Processing Technology, 100, 171-178.

https://doi.org/10.1016/S0924-0136(99)00472-0

[8] Fujiia, T., Tohgoa, K., Suzukib, Y., Yamamotoc, T., Shimamuraa, Y. and Ojimad, Y. (2011) Fatigue Properties of Spot Welded and Spot Weld-Bonded Joints of Steel Sheet. Procedia Engineering, 10, 1075-1080.

https://doi.org/10.1016/j.proeng.2011.04.177

[9] Almeida, F.J.S.D., Campilho, R.D.S.G., Silva, F.J.G. and Marques, G.P. (2018) Development of a Numerical Methodology for Strength Prediction of Weld-Bonded Joints. Procedia Manufacturing, 17, 713-720.

https://doi.org/10.1016/j.promfg.2018.10.121 